arthritis. Histological analysis of hind paws revealed an increase in synovial inflammation, bone erosion and cartilage damage in the CA treated animals compared to the Placebo group. No changes in joint pathology were observed in the UDCA or norUDCA treated group. Despite differences in absolute numbers of infiltrating cells, immunhistochemical staining for cell specific markers demonstrated no markedly changes in the distribution of neutrophils, macrophages, T- and B-lymphocytes within inflammatory synovial tissue. Similar amounts of total anti-collagen IgG and its subtypes IgG2 and $\operatorname{IgG} 2 \mathrm{c}$ were found in all treatment groups. Moreover, FACS analysis of lymph node cells revealed similar amounts of T-cells, $\mathrm{B}$-cells, dendritic cells and macrophages. In contrast, serum analyses revealed a trend toward increased IL- 6 levels suggesting that CA exacerbates IL-6 levels driving disease onset and severity in CIA model.

Conclusions Bile acid derivatives UDCA and norUDCA could not modulate collagen-induced arthritis. In contrast, cholic acid (CA) exacerbated the incidence and severity of inflammatory, erosive arthritis in this model. Thus, promising anti-inflammatory and anti-fibrotic agents for cholangiopathies have therefore no beneficial effects in an experimental arthritis model.

\section{A4.7 EVALUATION OF TISSUE INFLAMMATION AND ADIPOKINE EXPRESSION IN GASTRIC AND LUNG TISSUE OF SYSTEMIC SCLEROSIS (SSC) PATIENTS}

doi:10.1136/annrheumdis-2013-203217.7

\begin{abstract}
${ }^{1} \mathrm{~N}$ Lepper, ${ }^{1} \mathrm{M}$ Peters,,${ }^{1,2} \mathrm{M}$ Vasile, ${ }^{3} \mathrm{E}$ Roeb, ${ }^{4} \mathrm{~A}$ Günther, ${ }^{1} \mathrm{U}$ Müller-Ladner, 'E Neumann. 'Dept Internal Medicine and Rheumatology, Justus-Liebig-University Gießen, Kerckhoff Klinik, Bad Nauheim, Germany; ${ }^{2}$ Dept of Clinic and Medical Therapy, Div Rheumatology, Sapienza, University of Rome, Italy; Internal Medicine/Gastroenterology, University of Gießen, Gießen, Germany; ${ }^{4}$ Internal Medicine/Pneumology, University of Gießen, Gießen, Germany
\end{abstract}

Background Adipokines are bioactive substances secreted by adipose tissue and also by different resident cells. Adipokines are known to have influence on metabolism but there is inceasing evidence of the immunomodulatory role of different adipokines including adiponectin, visfatin/PBEF and resistin. For example, these adipokines are strongly expressed in synovial tissue of RA patients compared to controls. Since little is known about the role of adipokines in other rheumatic diseases including SSc, we analysed the expression of the adipokines adiponectin, resistin and visfatin in SSc organ involvement, specifically in gastric and lung tissue of SSc and control patients.

Methods Gastric samples from SSc $(n=9)$ and gastritis $(n=12)$ patients were examined for the expression of adiponectin, visfatin and resistin and also for the immune cell markers CD4, CD8 and CD68 by histology and immunohistochemistry. First, sections from each tissue were examined by hematoxilin/eosin (HE). Thereafter, lung samples from SSc $(n=2)$, IPF (idiopathic pulmonary fibrosis, $\mathrm{n}=8)$, and healthy controls $(\mathrm{n}=8)$ were examined by immunhistochemistry for the expression of adiponectin, visfatin, resistin and surfactant-protein C (SPC). Immune cell and adipokine expression was analysed qualitatively in gastric samples and semiqualitatively in lung samples.

Results The numbers of $\mathrm{CD}^{+}$and $\mathrm{CD}^{+}{ }^{+} \mathrm{T}$-cells in the gastric wall were comparable in SSc $(22.1 \pm 7.3$ resp. $15.3 \pm 2.2)$ and gastritis $(23.8 \pm 3.3$ resp. $15.3 \pm 4.0)$ patients. SSc patients showed a higher number of $\mathrm{CD} 68^{+}$immune cells compared to gastritis patients $(13.3 \pm 2.2$ versus $6.8 \pm 1.9)$, reaching statistical significance $(p=0.036)$. Visfatin and resistin expression in the gastric wall was present in most patients from both groups without significant difference. Gastric adiponectin expression was decreased significantly in SSc compared to gastritis patients $(p=0.049)$ being only present in SSc microvasculature if at all. Adiponectin expression was positively correlated to the number of $\mathrm{CD} 4^{+} \mathrm{T}$-cells and inversely correlated to the number of $\mathrm{CD} 68^{+}$immune cells. Lung samples of healthy and fibrotic (SSc and IPF) tissue showed a intermediate or strong visfatin and resistin expression. The pattern of resistin and visfatin expression was similar in all tissues, being cell-associated and present among bronchial epithel and immune cells, especially within lymphoid aggregates. Adiponectin was expressed in vessels and lung parenchyma, but not by immune cells. Again, adiponectin was decreased significantly within fibrotic lung tissue from SSc and IPF patients compared to healthy controls ( $p<0.0001)$.

Conclusions T-cell involvement in gastritis appears to be linked to inflammation including SSc even prior to onset of fibrosis, potentially enhanced by local macrophages and supported by resistin and visfatin. In contrast, the strong decrease of adiponectin in SSc gastric tissue and SSc and IPF lung tissue supports the idea of a role in fibrosis. Taken together, adipokines appear to be involved in distinct mechanisms of SSc and IPF pathophysiology.

The study was funded by the LOEWE initiative UGMLC: Universities of Giessen \& Marburg Lung Center.

\section{A4.8 GDF15, A MARKER OF LUNG INVOLVEMENT IN SYSTEMIC SCLEROSIS, IS INVOLVED IN ALTERED CYTOKINE SECRETION BY FIBROBLASTS, BUT DOES NOT IMPAIR FIBROSIS DEVELOPMENT}

doi:10.1136/annrheumdis-2013-203217.8

Stijn Lambrecht, Vanessa Smith, Filip De Keyser, Dirk Elewaut. Department of Rheumatology, Ghent University

Objective Systemic sclerosis is a progressive connective tissue involving autoimmune processes. It is generally known that members of the TGF- $\beta$ superfamily are involved in the regulation of connective tissue metabolism in systemic sclerosis (SSc), but also in regulating the immune system. Growth differentiation factor 15 is a distant member of this TGF- $\beta$ family. We aim to evaluate the role of GDF15 in SSc-pathogenesis.

Methods A longitudinal prospective cohort of SSc patients was screened for GDF15 serum levels by ELISA and associations with disease activity and tissue damage were analysed. Moreover, in vitro stimulation experiments were performed in lung fibroblasts. The role of GDF15 in fibrosis development in vivo was evaluated by performing the bleomycin lung fibrosis model in GDF15 deficient mice.

Results Serum samples from a cohort of 122 patients were screened for GDF15 levels. An increase in GDF15 levels was observed in patients classified as limited SSc, limited cutaneous SSc and diffuse SSc. Moreover, GDF15 serum levels highly correlated with disease activity and clinical symptoms of lung fibrosis. This was also mimicked in the bleomycin mouse model of SSc. Here, bleomycin exposed animals displayed elevated expression levels of GDF15 in lung tissue. Isolated lung fibroblast of GDF15 deficient mice showed reduced induction of IL6 and CCL2 upon bleomycin stimulation compared to wild-type littermates. Both, IL6 and CCL2, are involved in recruitment and activation of the immune system. Surprisingly, no differences in end-stage fibrosis development were observed between wild-type and GDF15 deficient animals after bleomycin injection.

Conclusions An intriguing profile of GDF15 serum levels was found in SSc patients. GDF15 expression is induced during fibrosis development. We hypothesise that the altered GDF15 expression by lung fibroblast may contribute to distorted interaction between the immune system and the stromal connective tissue. From our data it is clear that this protein may participate in fibrosis initiation, but is not indispensable in the course of fibrosis development in vivo. 